\title{
THE INVERSE PROBLEM OF THE CALCULUS OF VARIATIONS IN HIGHER SPACE*
}

\author{
BY
}

DAVID R. DAVIS

\section{INTRODUCTION}

The chief problem of this paper is to determine the integrand $f$ for an integral $I$ of the form

$$
I=\int_{x_{1}}^{x_{2}} f\left(x, y, z, y^{\prime}, z^{\prime}\right) d x
$$

whose Euler equations are

$$
H\left(x, y, z, y^{\prime}, z^{\prime}, y^{\prime \prime}, z^{\prime \prime}\right)=0, \quad K\left(x, y, z, y^{\prime}, z^{\prime}, y^{\prime \prime}, z^{\prime \prime}\right)=0 .
$$

The problem is impossible unless $H$ and $K$ have special properties. In determining these properties it is found to be recessary and sufficient that the equations of variation,

$$
\begin{aligned}
& H_{y} u+H_{z} v+H_{y^{\prime}} u^{\prime}+H_{z^{\prime}} v^{\prime}+H_{y^{\prime}} u^{\prime \prime}+H_{z^{\prime}} v^{\prime \prime}=0 \\
& K_{y} u+K_{z} v+K_{y^{\prime}} u^{\prime}+K_{z^{\prime}} v^{\prime}+K_{y^{\prime}} u^{\prime \prime}+K_{z^{\prime}} v^{\prime \prime}=0
\end{aligned}
$$

of $H=K=0$ be a self-adjoint system.

In $\$ I$ systems of adjoint differential equations of the second order are discussed. The unique existence of an adjoint system is proved and necessary and sufficient conditions for a given system of the second order to be selfadjoint are established.

Characteristic properties of the differential equations of the calculus of variations are derived in $\S I I$. It is here shown that if two equations $H=K=0$ are to be the Euler equations for an integral $I$ of the form given above they must have their equations of variation self-adjoint.

The sufficiency of this property is established in \$III. Necessary and sufficient conditions for the equations of variation of the system $H=K=0$ to be self-adjoint are given. Finally, the most general form of the integrand $f$ for the integral $I$ whose Euler equations are the equations $H=K=0$ is determined.

* Presented to the Society, San Francisco Section, June 18, 1927, under the titles Characteristic properties of differential equations of the calculus of variations, Integrals whose extremals have given differential equations, and $A$ method of determining an integral $I$ whose extremals are a given family of curves; received by the editors June 1, 1927. 
The problem of finding an integral whose extremals are a given family of curves is treated in §IV. Necessary and sufficient conditions for two given equations of the form

$$
y^{\prime \prime}=F\left(x, y, z, y^{\prime}, z^{\prime}\right), \quad \quad z^{\prime \prime}=G\left(x, y, z, y^{\prime}, z^{\prime}\right)
$$

to have solutions $y=y(x), z=z(x)$ which represent the extremals for an integral $I$ of the form above are derived. Also the possibility of determining linear combinations of two given equations $y^{\prime \prime}=F, z^{\prime \prime}=G$, which shall have equations of variation that are self-adjoint, is discussed.

In the last three sections special examples are treated. In $\S \mathrm{V}$ is found the most general type of integral whose extremals are straight lines. In $\S V I$ is treated the problem of finding integrals whose extremals are semicircles orthogonal to the $x y$-plane and finally, in §VII, that of determining integrals whose extremals are catenaries orthogonal to the $x y$-plane.*

\section{SELF-ADJOINT SYSTEMS OF LINEAR DIFFERENTIAL EXPRESSIONS OF THE SECOND ORDER}

The properties which characterize a self-adjoint system of differential expressions of the second order are needed in the discussion of the following sections. For convenience of reference they are developed here. $\dagger$

Consider the system of differential expressions

$$
J_{i}(u)=A_{i k}(x) u_{k}+B_{i k}(x) u_{k}^{\prime}+C_{i k}(x) u_{k}^{\prime \prime} \quad(i=1, \cdots, n),
$$

in the $n$ variables $u_{k}(k=1, \cdots, n)$. In this and following expressions the notation of tensor analysis is used, that is, whenever two subscripts are alike in a term, say of the form $A_{i k} u_{k}$, then the expression represents a sum with respect to the repeated index. The use of accents or primes will always indicate the total derivative of the function with respect to the independent variable.

A system $K_{i}(u)$ of the same form as (1) and satisfying with $J_{i}(u)$ an equation

$$
u_{i} J_{i}(v)-v_{i} K_{i}(u)=\frac{d M}{d x},
$$

* The writer wishes to take this opportunity to thank Dr. Bliss of Chicago for his advice and valuable suggestions during the preparation of this paper.

$\dagger$ See J. Kürschak, Die Existenzbedingungen des verallgemeinerten kinetischen Potentials, Mathematische Annalen, vol. 62 (1906), p. 148. Also G. Frobenius, Über adjungierte lineare Differentialausdriicke, Journal für Mathematik, vol. 85 (1878), p. 207. 
where $M$ is a function of $x, u_{i}, v_{k}$ and their derivatives, is called an adjoint system of $J_{i}(u)$.

It is easily proved that every system $J_{i}(u)$ has one and only one adjoint system $K_{i}(u)$. For from (1), we have

$$
\begin{aligned}
u_{i} J_{i}(v)= & u_{i} A_{i k} v_{k}+u_{i} B_{i k} v_{k}^{\prime}+u_{i} C_{i k} v_{k}^{\prime \prime} \\
= & u_{i} A_{i k} v_{k}+\frac{d}{d x}\left(u_{i} B_{i k} v_{k}\right)+\frac{d}{d x}\left[\left(u_{i} C_{i k}\right) v_{k}^{\prime}-\left(u_{i} C_{i k}\right)^{\prime} v_{k}\right] \\
& \quad-\left(u_{i} B_{i k}\right)^{\prime} v_{k}+\left(u_{i} C_{i k}\right)^{\prime \prime} v_{k}, \\
= & {\left[u_{i} A_{i k}-\left(u_{i} B_{i k}\right)^{\prime}+\left(u_{i} C_{i k}\right)^{\prime \prime}\right] v_{k} } \\
& +\frac{d}{d x}\left[u_{i} B_{i k} v_{k}+\left(u_{i} C_{i k}\right) v_{k}^{\prime}-\left(u_{i} C_{i k}\right)^{\prime} v_{k}\right] .
\end{aligned}
$$

Hence, there exist a system $K_{i}(u)$ and a function $M$ which satisfy equation (2), namely,

$$
\begin{aligned}
K_{i}(u) & =u_{i} A_{i k}-\left(u_{i} B_{i k}\right)^{\prime}+\left(u_{i} C_{i k}\right)^{\prime \prime}, \\
M & =u_{i} B_{i k} v_{k}+\left(u_{i} C_{i k}\right) v_{k}^{\prime}-\left(u_{i} C_{i k}\right)^{\prime} v_{k} .
\end{aligned}
$$

Suppose there were two such systems; then we would have the two equations

which give

$$
\begin{aligned}
& u_{i} J_{i}(v)-v_{i} K_{i}(u)=\frac{d M}{d x}, \\
& u_{i} J_{i}(v)-v_{i} H_{i}(u)=\frac{d L}{d x},
\end{aligned}
$$

$$
v_{i}\left[H_{i}(u)-K_{i}(u)\right]=\frac{d}{d x}(M-L) .
$$

If we represent the coefficients of the $v_{i}$ for a fixed set $u_{i}(x)$ by the notations $\phi_{i}(x)$, then the integral

$$
\int_{x_{1}}^{x_{3}} v_{i} \phi_{i}(x) d x=M-\left.L\right|_{x_{1}} ^{x_{2}}
$$

is clearly independent of the path in the $x v$-plane. This can be so, however, if and only if the functions $\phi_{i}(x)$ are identically zero, which implies that for every set of functions $u_{i}(x)$

$$
H_{i}(u) \equiv K_{i}(u) .
$$

Thus the adjoint system $K_{i}(u)$ as defined by (2) is unique.

The $J_{i}(u)$ are said to be self-adjoint if the systems $J_{i}(u)$ and $K_{i}(u)$ are identical. Imposing this condition, we have by (1) and (3) 


$$
\begin{aligned}
A_{i k} u_{k}+B_{i k} u_{k}^{\prime}+C_{i k} u_{k}^{\prime \prime} \equiv u_{k} A_{k i}-\left(u_{k} B_{k i}\right)^{\prime}+\left(u_{k} C_{k i}\right)^{\prime \prime} \\
\equiv u_{k}\left(A_{k i}-B_{k i}^{\prime}+C_{k i}^{\prime \prime}\right)+u_{k}^{\prime}\left(2 C_{k i}^{\prime}-B_{k i}\right)+u_{k}^{\prime \prime} C_{k i} .
\end{aligned}
$$

Regarding these equations as identities in the $u_{k}$ and their derivatives and equating the corresponding coefficients, we arrive at the following theorem:

TheоREм I. Necessary and sufficient conditions that the system of differential expressions

$$
J_{i}(u)=A_{i k}(x) u_{k}+B_{i k}(x) u_{k}^{\prime}+C_{i k}(x) u_{k}^{\prime \prime} \quad(i=1, \cdots, n)
$$

shall be self-adjoint are

$$
C_{i k} \equiv C_{k i}, \quad B_{i k}+B_{k i} \equiv 2 C_{i k}^{\prime}, \quad A_{i k} \equiv A_{k i}-B_{k i}^{\prime}+C_{k i}^{\prime \prime} .
$$

II. Characteristic properties of differential equations OF THE CALCULUS OF VARIATIONS*

In the problem of the calculus of variations in a space of $n+1$ dimensions one seeks to minimize the integral

$$
I=\int_{x_{1}}^{x_{2}} f\left(x, y_{1}, \cdots, y_{n}, y_{1}^{\prime}, \cdots, y_{n}^{\prime}\right) d x
$$

in a class of arcs $y_{k}=y_{k}(x), x_{1} \leqq x \leqq x_{2}$, joining two given points. It is well known that if a minimum is to occur it is necessary that the first variation of the integral $I$ must vanish and that the second variation must be always positive or zero. The vanishing of the first variation implies that the EulerLagrange equations

$$
\frac{d}{d x} f_{y^{\prime}}-f_{y}=0
$$

must be satisfied along a minimizing arc. The subscripts $y$ and $y^{\prime}$ are used to represent $y_{i}$ and $y_{i}^{\prime}$ respectively. If the equations

$$
y_{i}=y_{i}(x, b) \quad(i=1, \cdots, n)
$$

define a one-parameter family of solutions of the Euler-Lagrange equations, then the functions

$$
u_{i}=y_{i b}(x, b)
$$

are called the variations of the family along the arc (6). Along this arc they must satisfy the so-called equations of variation

$$
J_{i}(u)=\frac{d}{d x}\left(f_{i^{\prime} k} u_{k}+f_{i^{\prime} k^{\prime}} u_{k}^{\prime}\right)-\left(f_{i k} u_{k}+f_{i k^{\prime}} u_{k}^{\prime}\right)=0
$$

\footnotetext{
* See J. Hadamard, Leçons sur le Calcul des Variations, p. 151.
} 
which are found by differentiating equations (5) with respect to the parameter $b$ which occurs in the arguments $y_{i}$ and $y_{i}^{\prime}$ of the function $f$. The subscripts of $f$ denote differentiation with respect to the corresponding $y$ 's.

Let the function $\omega$ be defined by the expression

$$
2 \omega=f_{i k} u_{i} u_{k}+2 f_{i k^{\prime}} u_{i} u_{k}^{\prime}+f_{i^{\prime} k^{\prime}} u_{i}^{\prime} u_{k}^{\prime}
$$

then it is readily verified that

$$
J_{i}(u)=\frac{d}{d x} \omega_{u^{\prime}}-\omega_{u} \quad\left(u^{\prime}=u_{i}^{\prime}, u=u_{i}\right) .
$$

It is now easy to show that the system $J_{i}(u)$ is self-adjoint.* According to the definition of $\$ I$ we must have

$$
u_{i} J_{i}(v)-v_{i} J_{i}(u)=\frac{d M}{d x} .
$$

With the aid of (7) we readily find that

$$
u_{i} J_{i}(v)-v_{i} J_{i}(u)=\frac{d}{d x}\left(u_{i} \omega_{v^{\prime}}-v_{i} \omega_{u^{\prime}}\right),
$$

in which the subscripts $v^{\prime}$ and $u^{\prime}$ represent $v_{i}^{\prime}$ and $u_{i}^{\prime}$ respectively. Therefore a set of differential equations which are the differential equations of the solutions of a problem of the calculus of variations must have the property indicated in the following theorem:

THEOREM II. If a given system of differential equations

$$
H_{i}\left(x, y_{1}, \cdots, y_{n}, y_{1}^{\prime}, \cdots, y_{n}^{\prime}, y_{1}^{\prime \prime}, \cdots, y_{n}^{\prime \prime}\right)=0 \quad(j=1, \cdots, n)
$$

is to be the system of differential equations of the solutions of the problem of minimizing the integral I, of the type indicated above, then their equations of variation

$$
H_{j i} u_{i}+H_{j i^{\prime}} u_{i}^{\prime}+H_{j i^{\prime}} u_{i}^{\prime \prime}=0
$$

must be a self-adjoint system along every curve $y_{i}=y_{i}(x)$.

III. INTEGRALS WHOSE EXTREMALS HAVE GIVEN DIFFERENTIAL EQUATIONS

Consider the equations

$$
H\left(x, y, z, y^{\prime}, z^{\prime}, y^{\prime \prime}, z^{\prime \prime}\right)=0, \quad K\left(x, y, z, y^{\prime}, z^{\prime}, y^{\prime \prime}, z^{\prime \prime}\right)=0,
$$

whose solutions have the form

$$
y=y(x), \quad z=z(x)
$$

* It is of course understood that the argument here is concerning the Euler equations as they stand and not an equivalent system. 
with the derivatives $y^{\prime}, z^{\prime}, y^{\prime \prime}, z^{\prime \prime}$. It will be shown, upon the assumption that the equations of variation of $H=K=0$ form a self-adjoint system, that a function $f\left(x, y, z, y^{\prime}, z^{\prime}\right)$ can be determined such that the equations (8) are the differential equations of the solutions of the problem of minimizing the integral

$$
I=\int_{x_{1}}^{x_{2}} f\left(x, y, z, y^{\prime}, z^{\prime}\right) d x
$$

The equations of variation of the given system (8) are

$$
\begin{aligned}
& H_{y} u+H_{z} v+H_{y^{\prime}} u^{\prime}+H_{z^{\prime}} v^{\prime}+H_{y^{\prime \prime}} u^{\prime \prime}+H_{z^{\prime}} v^{\prime \prime}=0, \\
& K_{y} u+K_{z} v+K_{y^{\prime}} u^{\prime}+K_{z^{\prime}} v^{\prime}+K_{y^{\prime}} u^{\prime \prime}+K_{z^{\prime}} v^{\prime \prime}=0 .
\end{aligned}
$$

If these equations are a self-adjoint system, then by Theorem I, we have the following relations:

$$
\begin{gathered}
H_{z^{\prime \prime}}=K_{y^{\prime \prime}}, \quad H_{y^{\prime}}=H_{y^{\prime \prime}}^{\prime}, \quad K_{z^{\prime}}=K_{z^{\prime \prime}}^{\prime}, \\
H_{z^{\prime}}+K_{y^{\prime}}=2 H_{z^{\prime \prime}}^{\prime \prime}=2 K_{y^{\prime \prime}}^{\prime}, \\
K_{y}=H_{z}-H_{z^{\prime}}^{\prime}+H_{z^{\prime \prime}}^{\prime \prime}, \quad H_{z}=K_{y}-K_{y^{\prime}}^{\prime}+K_{y^{\prime \prime}}^{\prime \prime},
\end{gathered}
$$

which must be identities in $x, y, z, y^{\prime}, z^{\prime}, y^{\prime \prime}, z^{\prime \prime}, y^{\prime \prime \prime}, z^{\prime \prime \prime}$. The last of equations (11) is a consequence of the preceding relations. For we may solve the fifth equation for $H_{z}$ and make use of relations (1) and (4) of (11). Then

$$
\begin{aligned}
H_{z} & =K_{y}+H_{z^{\prime}}^{\prime}-H_{z^{\prime \prime}}^{\prime \prime}, \\
& =K_{y}+2 K_{y^{\prime \prime}}^{\prime \prime}-K_{y^{\prime}}^{\prime}-K_{y^{\prime \prime}}^{\prime \prime},
\end{aligned}
$$

which gives the last of equations (11).

In the second and third of relations (11) terms in $y^{\prime \prime \prime}$ and $z^{\prime \prime \prime}$ do not occur in the first members. It is thus evident upon expanding the second members that the derivatives $H_{y^{\prime \prime} y^{\prime \prime}} ; H_{y^{\prime \prime} z^{\prime \prime}} ; K_{z^{\prime \prime} y^{\prime \prime}} ; K_{z^{\prime \prime} z^{\prime \prime}}$ all vanish identically, and hence, that the given equations (8) are linear in $y^{\prime \prime}$ and $z^{\prime \prime}$. Also the first equation of (11) asserts that the coefficient of $z^{\prime \prime}$ in $\left(8_{1}\right)$ is equal to the coefficient of $y^{\prime \prime}$ in $\left(8_{2}\right)$. Therefore the given equations may be expressed in the form

$$
\begin{aligned}
& H=M+P y^{\prime \prime}+Q z^{\prime \prime}=0, \\
& K=N+Q y^{\prime \prime}+R z^{\prime \prime}=0,
\end{aligned}
$$

where $M, N, P, Q, R$ are functions of $x, y, z, y^{\prime}, z^{\prime}$.

In view of the first of relations (11) and upon subtracting the fifth from the last, one obtains 


$$
2\left(H_{z}-K_{y}\right)=H_{z^{\prime}}^{\prime}-K_{y^{\prime}}^{\prime}=\frac{d}{d x}\left(H_{z^{\prime}}-K_{y^{\prime}}\right) .
$$

Expanding the second member of this equation and noting that terms in $y^{\prime \prime \prime}$ and $z^{\prime \prime \prime}$ do not occur on the left, we have

$$
H_{z^{\prime} y^{\prime \prime}}-K_{y^{\prime} y^{\prime \prime}}=0, \quad H_{z^{\prime} z^{\prime \prime}}-K_{y^{\prime} z^{\prime \prime}}=0 .
$$

Hence, by $\left(11_{1}\right)$ each of the functions $H$ and $K$ must satisfy the conditions

$$
H_{y^{\prime} z^{\prime \prime}}=H_{y^{\prime \prime} z^{\prime}}, \quad K_{y^{\prime} z^{\prime \prime}}=K_{y^{\prime \prime} z^{\prime}},
$$

which in terms of the notation in equations (12) become

$$
P_{z^{\prime}}=Q_{y^{\prime}}, \quad Q_{z^{\prime}}=R_{y^{\prime}} .
$$

These properties of the given functions $B$ and $K$ are the necessary conditions of integrability which are later required in the determination of the integrand $f$.

With the aid of (12) and (13) the second, third and fourth of the selfadjoint relations (11) may be written in the form

$$
\begin{aligned}
M_{y^{\prime}} & =P_{x}+P_{y} y^{\prime}+P_{z} z^{\prime}, \\
N_{z^{\prime}} & =R_{x}+R_{y} y^{\prime}+R_{z} z^{\prime}, \\
M_{z^{\prime}}+N_{y^{\prime}} & =2\left(Q_{x}+Q_{y} y^{\prime}+Q_{z} z^{\prime}\right) .
\end{aligned}
$$

Since the last two of relations (11) are equivalent, the use of either together with equations (12) will give

$$
M_{z}-N_{y}=\left(Q_{y}-P_{z}\right) y^{\prime \prime}+\left(R_{y}-Q_{z}\right) z^{\prime \prime}+\frac{1}{2}\left(M_{z^{\prime}}-N_{y^{\prime}}\right)^{\prime} .
$$

If this equation is to be an identity in the variables involved, we must have

$$
\begin{aligned}
Q_{y}-P_{z} & =-\frac{1}{2}\left(M_{z^{\prime} y^{\prime}}-N_{y^{\prime} y^{\prime}}\right), \\
R_{y}-Q_{z} & =-\frac{1}{2}\left(M_{z^{\prime} z^{\prime}}-N_{y^{\prime} z^{\prime}}\right), \\
M_{z}-N_{y} & =\frac{1}{2}\left[\left(M_{z^{\prime}}-N_{y^{\prime}}\right)_{x}+\left(M_{z^{\prime}}-N_{y^{\prime}}\right)_{y} y^{\prime}+\left(M_{z^{\prime}}-N_{y^{\prime}}\right)_{z} z^{\prime}\right] .
\end{aligned}
$$

The first two of these relations may be obtained without difficulty from (14) and (13). But the third involves an independent relation which must be satisfied by the functions $M$ and $N$. We can now state the following theorem:

THEOREM III. If a system of equations

$$
H\left(x, y, z, y^{\prime}, z^{\prime}, y^{\prime \prime}, z^{\prime \prime}\right)=0, \quad K\left(x, y, z, y^{\prime}, z^{\prime}, y^{\prime \prime}, z^{\prime \prime}\right)=0
$$

is to have equations of variation

$$
\begin{aligned}
& H_{y} u+H_{z} v+H_{y^{\prime}} u^{\prime}+H_{z^{\prime}} v^{\prime}+H_{y^{\prime \prime}} u^{\prime \prime}+H_{z^{\prime \prime}} v^{\prime \prime}=0, \\
& K_{y} u+K_{z} v+K_{y^{\prime}} u^{\prime}+K_{z^{\prime}} v^{\prime}+K_{y^{\prime}} u^{\prime \prime}+K_{z^{\prime \prime}} v^{\prime \prime}=0,
\end{aligned}
$$


which are self-adjoint along every curve $y=y(x), z=z(x)$, then it must have the form

$$
\begin{aligned}
& H=M\left(x, y, z, y^{\prime}, z^{\prime}\right)+P\left(x, y, z, y^{\prime}, z^{\prime}\right) y^{\prime \prime}+Q\left(x, y, z, y^{\prime}, z^{\prime}\right) z^{\prime \prime}, \\
& K=N\left(x, y, z, y^{\prime}, z^{\prime}\right)+Q\left(x, y, z, y^{\prime}, z^{\prime}\right) y^{\prime \prime}+R\left(x, y, z, y^{\prime}, z^{\prime}\right) z^{\prime \prime},
\end{aligned}
$$

where $M, N, P, Q, R$ satisfy the following relations:

$$
\begin{aligned}
P_{z^{\prime}} & =Q_{y^{\prime}}, \quad Q_{z^{\prime}}=R_{y^{\prime}}, \\
M_{y^{\prime}} & =P_{x}+P_{y} y^{\prime}+P_{z} z^{\prime}, \quad N_{z^{\prime}}=R_{x}+R_{y} y^{\prime}+R_{z} z^{\prime}, \\
M_{z^{\prime}}+N_{y^{\prime}} & =2\left(Q_{x}+Q_{y} y^{\prime}+Q_{z} z^{\prime}\right), \\
M_{z}-N_{y} & =\frac{1}{2}\left[\left(M_{z^{\prime}}-N_{y^{\prime}}\right)_{x}+\left(M_{z^{\prime}}-N_{y^{\prime}}\right)_{y} y^{\prime}+\left(M_{z^{\prime}}-N_{y^{\prime}}\right)_{z} z^{\prime}\right],
\end{aligned}
$$

identically in $x, y, z, y^{\prime}, z^{\prime}$.

If a system of equations $H=K=0$ has the form indicated in the last theorem with coefficients satisfying (16), then it is possible to determine the integrand of the integral $I$ in (9) to an arbitrary function of $x, y, z$ so that $H=K=0$ are the equations of its minimizing curves. Let us first determine a function $g\left(x, y, z, y^{\prime}, z^{\prime}\right)$ satisfying the relations

$$
g_{y^{\prime} y^{\prime}}=P, \quad g_{y^{\prime} z^{\prime}}=Q, \quad g_{z^{\prime} z^{\prime}}=R .
$$

It is possible to do this since the conditions for integrability of the system (17) are the first two of the relations (16) for self-adjointness. The function $g$ will then be determined by the integral

$$
g=\int_{y_{0}^{\prime} z_{0^{\prime}}}^{y^{\prime} z^{\prime}} M d y^{\prime}+L d z^{\prime}
$$

where the functions $M$ and $L$ represent the integrals

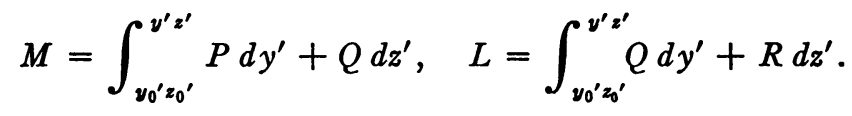

The first two of relations (16) also insure that the above line integrals are independent of the path of integration. It is evident that if $g$ is a particular solution of the system (17) then the most general one is given by the formula

$$
f=g+a+b y^{\prime}+c z^{\prime}
$$

where $a, b, c$ are constants with respect to the integrations for $y^{\prime}$ and $z^{\prime}$ and therefore functions of $x, y, z$.

If the given equations (8) are identical with the Euler-Lagrange equations for the integral (9), then the equations 


$$
\frac{d}{d x} f_{y^{\prime}}-f_{y}=M+P y^{\prime \prime}+Q z^{\prime \prime}, \frac{d}{d x} f_{z^{\prime}}-f_{z}=N+Q y^{\prime \prime}+R z^{\prime \prime}
$$

must be identities in $x, y, z, y^{\prime}, z^{\prime}, y^{\prime \prime}, z^{\prime \prime}$.

The value of $f$ given in (18) must now satisfy equations (19). Inserting this value of $f$, we get

$$
\begin{aligned}
& \frac{d}{d x}\left(g_{y^{\prime}}+b\right)-\left(g_{y}+a_{y}+b_{y} y^{\prime}+c_{y} z^{\prime}\right)=M+P y^{\prime \prime}+Q z^{\prime \prime}, \\
& \frac{d}{d x}\left(g_{z^{\prime}}+c\right)-\left(g_{z}+a_{z}+b_{z} y^{\prime}+c_{z} z^{\prime}\right)=N+Q y^{\prime \prime}+R z^{\prime \prime},
\end{aligned}
$$

which reduce to

$$
\begin{aligned}
& \left(b_{x}-a_{y}\right)+\left(b_{z}-c_{y}\right) z^{\prime}=M+g_{y}-g_{y^{\prime} x}-g_{y^{\prime} y} y^{\prime}-g_{y^{\prime} z} z^{\prime}, \\
& \left(c_{x}-a_{z}\right)+\left(c_{y}-b_{z}\right) y^{\prime}=N+g_{z}-g_{z^{\prime} x}-g_{z^{\prime} y} y^{\prime}-g_{z^{\prime}} z^{\prime} .
\end{aligned}
$$

If these equations are to be identities in $x, y, z, y^{\prime}, z^{\prime}$ then the second members must be independent of $y^{\prime}$ and $z^{\prime}$ respectively, while the expression on the right in the first equation must be linear in $z^{\prime}$ and that of the second equation linear in $y^{\prime}$. If the second member of the first equation is differentiated once with respect to $y^{\prime}$, the result by the third of relations (16) is identically zero. The second derivative of this same expression with respect to $z^{\prime}$ is readily shown to be identically zero by means of the fifth of (16) and the first two of (15), which are direct consequences of $\left(16_{3}\right)$ and $\left(16_{4}\right)$. One proceeds similarly in the case of the second equation. We thus have the required condition that $a, b$ and $c$ are functions of $x, y, z$ alone.

From (20) one obtains the relations

$$
\begin{aligned}
& c_{y}-b_{z}=g_{y^{\prime} z}-g_{z^{\prime}}-\frac{1}{2}\left(M_{z^{\prime}}-N_{y^{\prime}}\right), \\
& a_{z}-c_{x}=-N-g_{z}+g_{z^{\prime} x}+g_{y^{\prime} z} y^{\prime}+g_{z^{\prime}} z^{\prime}-\frac{1}{2}\left(M_{z^{\prime}}-N_{y^{\prime}}\right) y^{\prime}, \\
& b_{x}-a_{y}=M+g_{y}-g_{y^{\prime} x}-g_{y^{\prime} y} y^{\prime}-g_{z^{\prime}} z^{\prime}-\frac{1}{2}\left(M_{z^{\prime}}-N_{y^{\prime}}\right) z^{\prime} .
\end{aligned}
$$

The first one of these equations is found by differentiating the first or second equation of (20) with respect to $y^{\prime}$ or $z^{\prime}$ respectively and employing $\left(16_{5}\right)$. The substitution of the value so found for $c_{y}-b_{z}$ in equations (20) gives the second and third of (21). The system (21) has solutions for $a, b, c$ if and only if the second members, which from their derivation are independent of $\boldsymbol{y}^{\prime}$ and $z^{\prime}$, satisfy the equation

$$
\frac{\partial p}{\partial x}+\frac{\partial q}{\partial y}+\frac{\partial r}{\partial z} \equiv 0
$$


identically in $x, y, z$; where $p, q, r$ represent the second members respectively. Performing the differentiation as indicated by (22) one finds that the terms in the derivatives of $g$ cancel in pairs and that the resulting expression is identically zero by the last of relations (16).

Let $a, b, c$ be a particular set of solutions of (21); then the most general solution will be

$$
a+\alpha, \quad b+\beta, \quad c+\gamma,
$$

where $\alpha, \beta, \gamma$ must satisfy the equations

$$
\gamma_{y}-\beta_{z}=0, \quad \alpha_{z}-\gamma_{x}=0, \quad \beta_{x}-\alpha_{y}=0
$$

which are necessary and sufficient conditions for

$$
\alpha+\beta y^{\prime}+\gamma z^{\prime}
$$

to be the total derivative of a function $t(x, y, z)$.

The function $f$ of (18) can now be expressed in the form

$$
f=g+a+b y^{\prime}+c z^{\prime}+\frac{d t}{d \lambda}
$$

where $a, b, c$ are a particular set of solutions of (21), and $t$ is an arbitrary function of $(x, y, z)$. Hence, we have

THEOREM IV. If two equations of the form

$$
H\left(x, y, z, y^{\prime}, z^{\prime}, y^{\prime \prime}, z^{\prime \prime}\right)=0, \quad K\left(x, y, z, y^{\prime}, z^{\prime}, y^{\prime \prime}, z^{\prime \prime}\right)=0
$$

have equations of variation

$$
\begin{aligned}
& H_{y} u+H_{z} v+H_{y^{\prime}} u^{\prime}+H_{z^{\prime}} v^{\prime}+H_{y^{\prime \prime}} u^{\prime \prime}+H_{z^{\prime}} \cdot v^{\prime \prime}=0, \\
& K_{y} u+K_{z} v+K_{y^{\prime}} u^{\prime}+K_{z^{\prime}} v^{\prime}+K_{y^{\prime \prime}} u^{\prime \prime}+K_{z^{\prime}, v^{\prime \prime}}=0,
\end{aligned}
$$

which form a self-adjoint system along every curve $y=y(x), z=z(x)$, then there is always an integral of the form

$$
I=\int_{x_{1}}^{x_{2}} f\left(x, y, z, y^{\prime}, z^{\prime}\right) d x
$$

having the equations $B=K=0$ as its Euler equations. The most general such integral has an integrand

$$
f=g\left(x, y, z, y^{\prime}, z^{\prime}\right)+a(x, y, z)+b(x, y, z) y^{\prime}+c(x, y, z) z^{\prime}+\frac{d}{d x} t(x, y, z)
$$

where $g$ is a particular solution of (17); $a, b, c$ are a particular solution of (21); and $t$ is an arbitrary function of $x, y, z$. 
IV. A METHOD OF DETERMINING AN INTEGRAL $I$ WHOSE EXTREMALS ARE A GIVEN FAMILY OF CURVES

Consider the four-parameter family of arcs

$$
y=y(x, a, b, c, d), \quad z=z(x, a, b, c, d) .
$$

Differentiation with respect to $x$ gives

$$
\begin{aligned}
y^{\prime} & =y_{x}(x, a, b, c, d), & z^{\prime} & =z_{x}(x, a, b, c, d), \\
y^{\prime \prime} & =y_{x x}(x, a, b, c, d), & z^{\prime \prime} & =z_{x x}(x, a, b, c, d) .
\end{aligned}
$$

If $a, b, c, d$ are eliminated from the above equations one obtains two equations of the form

$$
y^{\prime \prime}=F\left(x, y, z, y^{\prime}, z^{\prime}\right), \quad z^{\prime \prime}=G\left(x, y, z, y^{\prime}, z^{\prime}\right) .
$$

The general solutions of these equations are (23) where $a, b, c, d$ are constants of integration.

If the equations (23) are to represent the extremal arcs of an integral $I$ of the form (9), then by Theorem II the functions $F$ and $G$ in (24) must be the solutions for $y^{\prime \prime}$ and $z^{\prime \prime}$, respectively, of the system of equations

$$
H\left(x, y, z, y^{\prime}, z^{\prime}, y^{\prime \prime}, z^{\prime \prime}\right)=0, \quad K\left(x, y, z, y^{\prime}, z^{\prime}, y^{\prime \prime}, z^{\prime \prime}\right)=0
$$

whose equations of variation are self-adjoint. Hence, there must exist a set of multipliers $P, Q_{1}, Q_{2}, R$ such that the equations

$$
\begin{aligned}
& P\left(y^{\prime \prime}-F\right)+Q_{1}\left(z^{\prime \prime}-G\right)=0, \\
& Q_{2}\left(y^{\prime \prime}-F\right)+R\left(z^{\prime \prime}-G\right)=0
\end{aligned}
$$

can be rendered identical with equations (12) in the previous section. It is evident from the form of (12) that the desired multipliers have $Q_{1}=Q_{2}$ and hence, that the functions $H$ and $K$ may be expressed in the following way:

$$
\begin{aligned}
& H=P y^{\prime \prime}+Q z^{\prime \prime}-(P F+Q G)=0, \\
& K=Q y^{\prime \prime}+R z^{\prime \prime}-(Q F+R G)=0 .
\end{aligned}
$$

The functions $M$ and $N$ of equations (12) now have the values

$$
M=-(P F+Q G), \quad N=-(Q F+R G) .
$$

Necessary and sufficient conditions for the system (25) to be self-adjoint may, therefore, be obtained from Theorem III. Evidently, the first two relations remain as in (16), namely, 


$$
P_{z^{\prime}}=Q_{y^{\prime}}, \quad Q_{z^{\prime}}=R_{y^{\prime}} .
$$

Making the substitution (26) in $\left(16_{3}\right),\left(16_{5}\right),\left(16_{4}\right)$ respectively, one obtains the following system:

$$
\begin{aligned}
P_{x}+P_{y} y^{\prime}+P_{z} z^{\prime} & =-(P F+Q G)_{y^{\prime}}, \\
Q_{x}+Q_{y} y^{\prime}+Q_{z} z^{\prime} & =-\frac{1}{2}\left[(P F+Q G)_{z^{\prime}}+(Q F+R G)_{y^{\prime}}\right], \\
R_{x}+R_{y} y^{\prime}+R_{z} z^{\prime} & =-(Q F+R G)_{z^{\prime}} .
\end{aligned}
$$

With the use of (27) these equations may be written in the form

$$
\begin{aligned}
P_{x}+P_{y} y^{\prime}+P_{z} z^{\prime}+P_{y^{\prime}} F+P_{z^{\prime}} G & =-\left(P F_{y^{\prime}}+Q G_{y^{\prime}}\right), \\
\text { (29) } Q_{x}+Q_{y} y^{\prime}+Q_{z} z^{\prime}+Q_{y^{\prime}} F+Q_{z^{\prime}} G & =-\frac{1}{2}\left(P F_{z^{\prime}}+Q G_{z^{\prime}}+Q F_{y^{\prime}}+R G_{y^{\prime}}\right), \\
R_{x}+R_{y} y^{\prime}+R_{z} z^{\prime}+R_{y^{\prime}} F+R_{z^{\prime}} G & =-\left(Q F_{z^{\prime}}+R G_{z^{\prime}}\right) .
\end{aligned}
$$

If in the last of equations (16) $M$ and $N$ are replaced by their values in (26), we get

(30) $(Q F+R G)_{y}-(P F+Q G)_{z} \equiv \frac{1}{2}\left[\left(Q F_{y^{\prime}}+R G_{y^{\prime}}-P F_{z^{\prime}}-Q G_{z^{\prime}}\right)_{x}\right.$

$$
\left.+\left(Q F_{y^{\prime}}+R G_{y^{\prime}}-P F_{z^{\prime}}-Q G_{z^{\prime}}\right)_{y} y^{\prime}+\left(Q F_{y^{\prime}}+R G_{y^{\prime}}-P F_{z^{\prime}}-Q G_{z^{\prime}}\right)_{z} z^{\prime}\right] \text {. }
$$

From the first two of (15), which were shown to be consequences of the first five of relations (16), arise by the substitution (26) the following equations:

$$
\begin{aligned}
& Q_{y}-P_{z}=\left(Q_{x}+Q_{y} y^{\prime}+Q_{z} z_{y^{\prime}}-\left(P_{x}+P_{y} y^{\prime}+P_{z} z^{\prime}\right)_{z^{\prime}},\right. \\
& R_{y}-Q_{z}=\left(R_{x}+R_{y} y^{\prime}+R_{z} z^{\prime}\right)_{y^{\prime}}-\left(Q_{x}+Q_{y} y^{\prime}+Q_{z} z_{z^{\prime}},\right.
\end{aligned}
$$

which by means of (27) and (28) reduce to

$$
\begin{aligned}
Q_{y}-P_{z}= & \frac{1}{2}\left[\left(P_{y^{\prime}} F_{z^{\prime}}+P F_{y^{\prime} z^{\prime}}+Q_{y^{\prime}} G_{z^{\prime}}+Q G_{y^{\prime} z^{\prime}}\right)\right. \\
& \left.-\left(Q_{y^{\prime}} F_{y^{\prime}}+Q F_{y^{\prime} y^{\prime}}+R_{y^{\prime}} G_{y^{\prime}}+R G_{y^{\prime} y^{\prime}}\right)\right], \\
R_{y}-Q_{z}= & \frac{1}{2}\left[\left(P_{z^{\prime}} F_{z^{\prime}}+P F_{z^{\prime} z^{\prime}}+Q_{z^{\prime}} G_{z^{\prime}}+Q G_{z^{\prime} z^{\prime}}\right)\right. \\
& \left.-\left(Q_{z^{\prime}} F_{y^{\prime}}+Q F_{y^{\prime} z^{\prime}}+R_{z^{\prime}} G_{y}+R G_{y^{\prime} z^{\prime}}\right)\right] .
\end{aligned}
$$

The expressions on the left are now replaced by those on the right, respectively, in the first member of (30) after the indicated differentiation is performed. After performing the indicated differentiation in the second member of (30) and employing relations (28), the result readily reduces to

$$
\begin{aligned}
& P\left[F_{z}+\frac{1}{4} F_{y^{\prime}} F_{z^{\prime}}+\frac{1}{4} F_{z^{\prime}} G_{z^{\prime}}-\frac{1}{2} F_{z^{\prime}}^{\prime}\right]+Q\left[\left(G_{z}-F_{y}\right)+\frac{1}{4}\left(G_{z^{\prime}}^{2}-F_{y^{\prime}}^{2}\right)\right. \\
& \left.\quad+\frac{1}{2}\left(F_{y^{\prime}}-G_{z^{\prime}}\right)^{\prime}\right]+R\left[-G_{y}-\frac{1}{4} G_{y^{\prime}} G_{z^{\prime}}-\frac{1}{4} F_{y^{\prime}} G_{y^{\prime}}+\frac{1}{2} G_{y^{\prime}}^{\prime}\right] \equiv 0,
\end{aligned}
$$

where it is understood that 


$$
\begin{aligned}
& F_{z^{\prime}}^{\prime} \equiv F_{z^{\prime} x}+F_{z^{\prime} y} y^{\prime}+F_{z^{\prime} z} z^{\prime}+F_{z^{\prime} y^{\prime}} F+F_{z^{\prime} z^{\prime}} G, \\
& \left(F_{y^{\prime}}-G_{z^{\prime}}\right)^{\prime} \equiv\left(F_{y^{\prime}}-G_{z^{\prime}}\right)_{x}+\left(F_{y^{\prime}}-G_{z^{\prime}}\right)_{y} y^{\prime}+\left(F_{y^{\prime}}-G_{z^{\prime}}\right)_{z^{\prime}} \\
& +\left(F_{y^{\prime}}-G_{z^{\prime}}\right)_{y^{\prime}} F+\left(F_{y^{\prime}}-G_{z^{\prime}}\right)_{z^{\prime}} G, \\
& G_{y^{\prime}}^{\prime} \equiv G_{y^{\prime} x}+G_{y^{\prime} z^{\prime}}+G_{y^{\prime} z} y^{\prime}+G_{y^{\prime} y^{\prime}} F+G_{y^{\prime} z^{\prime}} G \text {. }
\end{aligned}
$$

In view of the relations (27), (29), and (31) we have the following theorem:

TheOREM V. If the solutions $y=y(x), z=z(x)$ of

$$
y^{\prime \prime}=F\left(x, y, z, y^{\prime}, z^{\prime}\right), \quad z^{\prime \prime}=G\left(x, y, z, y^{\prime}, z^{\prime}\right)
$$

are to be the totality of extremal arcs for an integral of the form (9), then there must exist a set of multipliers $P, Q, R$ which are functions of $x, y, z, y^{\prime}, z^{\prime}$ such that the equations

$$
\begin{aligned}
& H=P\left(y^{\prime \prime}-F\right)+Q\left(z^{\prime \prime}-G\right)=0, \\
& K=Q\left(y^{\prime \prime}-F\right)+R\left(z^{\prime \prime}-G\right)=0
\end{aligned}
$$

have equations of variation which are self-adjoint along every arc $y=y(x)$, $z=z(x)$. Necessary and sufficient conditions for such multipliers to exist are

$$
\begin{gathered}
P_{z^{\prime}}=Q_{y^{\prime}}, \quad Q_{z^{\prime}}=R_{y^{\prime}}, \\
P_{x}+P_{y} y^{\prime}+P_{z} z^{\prime}+P_{y^{\prime}} F+P_{z^{\prime}} G=-\left(P F_{y^{\prime}}+Q G_{y^{\prime}}\right), \\
Q_{x}+Q_{y} y^{\prime}+Q_{z} z^{\prime}+Q_{y^{\prime}} F+Q_{z^{\prime}} G=-\frac{1}{2}\left(P F_{z^{\prime}}+Q G_{z^{\prime}}+Q F_{y^{\prime}}+R G_{y^{\prime}}\right), \\
R_{x}+R_{y} y^{\prime}+R_{z} z^{\prime}+R_{y^{\prime}} F+R_{z^{\prime}} G=-\left(Q F_{z^{\prime}}+R G_{z^{\prime}}\right), \\
P\left[F_{z}+\frac{1}{4} F_{y^{\prime}} F_{z^{\prime}}+\frac{1}{4} F_{z^{\prime}} G_{z^{\prime}}-\frac{1}{2} F_{z^{\prime}}^{\prime}\right]+Q\left[\left(G_{z}-F_{y}\right)+\frac{1}{4}\left(G_{z^{\prime}}^{2}-F_{y^{\prime}}^{2}\right)\right. \\
\left.+\frac{1}{2}\left(F_{y^{\prime}}-G_{z^{\prime}}\right)^{\prime}\right]+R\left[-G_{y}-\frac{1}{4} G_{y^{\prime}} G_{z^{\prime}}-\frac{1}{4} F_{y^{\prime}} G_{y^{\prime}}+\frac{1}{2} G_{y^{\prime}}^{\prime}\right] \equiv 0,
\end{gathered}
$$

which must be identities in $x, y, z, y^{\prime}, z^{\prime}$.

As is evident from the preceding discussion no criterion has been found whereby one can determine in advance whether the given functions $F$ and $G$ are solutions for $y^{\prime \prime}$ and $z^{\prime \prime}$, respectively, of a system of equations

$$
H\left(x, y, z, y^{\prime}, z^{\prime}, y^{\prime \prime}, z^{\prime \prime}\right)=0, \quad K\left(x, y, z, y^{\prime}, z^{\prime}, y^{\prime \prime}, z^{\prime \prime}\right)=0
$$

whose equations of variation are self-adjoint. If, however, the functions $F$ and $G$ are such that conditions (32) can be satisfied then the solutions of (24), $y=y(x), z=z(x)$, represent the extremal arcs for an integral $I$ of the form (9). Some difficulties have been encountered in attempting to satisfy conditions (32) and the writer has not been able to develop completely the theory for the general case. However, the following discussion together with 
the examples treated below will illustrate a method of procedure which may be employed with success in special cases.

The theory of partial differential equations assures us of the existence of solutions $P, Q, R$ of the system (29). To find these solutions let us introduce the new variables $x, a, b, c, d$ in place of $x, y, z, y^{\prime}, z^{\prime}$ by means of the equations

$$
\begin{array}{ll}
y=y(x, a, b, c, d), & y^{\prime}=y_{x}(x, a, b, c, d), \\
z=z(x, a, b, c, d), & z^{\prime}=z_{x}(x, a, b, c, d),
\end{array}
$$

which have solutions of the form

$$
\begin{array}{ll}
a=A\left(x, y, z, y^{\prime}, z^{\prime}\right), & c=C\left(x, y, z, y^{\prime}, z^{\prime}\right), \\
b=B\left(x, y, z, y^{\prime}, z^{\prime}\right), & d=D\left(x, y, z, y^{\prime}, z^{\prime}\right) .
\end{array}
$$

It is readily verified that every set of functions $P, Q, R$ of $x, a, b, c, d$ which satisfy (29), when $a, b, c, d$ are replaced by the expressions (34), must satisfy the equations

$$
\begin{aligned}
& \frac{d P}{d x}=-\left(P F_{y^{\prime}}+Q G_{y^{\prime}}\right), \\
& \frac{d Q}{d x}=-\frac{1}{2}\left(P F_{z^{\prime}}+Q G_{z^{\prime}}+Q F_{y^{\prime}}+R G_{y^{\prime}}\right), \\
& \frac{d R}{d x}=-\left(Q F_{z^{\prime}}+R G_{z^{\prime}}\right),
\end{aligned}
$$

and conversely. This follows because each of the functions (34) is a solution of the homogeneous equation of the type

$$
A_{x}+A_{y} y^{\prime}+A_{z} z^{\prime}+A_{y^{\prime}} F+A_{z^{\prime}} G \equiv 0 .
$$

In the equations (35) the variables $x, y, z, y^{\prime}, z^{\prime}$ are everywhere to be replaced by $x, a, b, c, d$ by means of the equations (33).

According to the theory of ordinary differential equations, if $P_{1}, Q_{1}, R_{1}$; $P_{2}, Q_{2}, R_{2} ; P_{3}, Q_{3}, R_{3}$ are any three independent particular solutions of the system (35), then every other solution can be expressed in the form

$$
\begin{aligned}
C_{1} P_{1}+C_{2} P_{2}+C_{3} P_{3} & =P(x, a, b, c, d), \\
C_{1} Q_{1}+C_{2} Q_{2}+C_{3} Q_{3} & =Q(x, a, b, c, d), \\
C_{1} R_{1}+C_{2} R_{2}+C_{3} R_{3} & =K(x, a, b, c, d),
\end{aligned}
$$

where $C_{1}, C_{2}, C_{3}$ are arbitrary functions of $a, b, c, d$. If $C_{1}, C_{2}, C_{8}$ are determined in any manner and the functions $a, b, c, d$ are replaced by their 
respective values in (34) the resulting values of $P, Q, R$ are solutions of the system (29). Conversely, every solution of the system (29) can be so obtained.

The functions $C_{1}, C_{2}, C_{3}$ must be determined so that the remaining conditions for self-adjointness of Theorem V, namely (27) and (31), shall be satisfied. The possibility of satisfying these conditions by a suitable choice of the arbitrary functions $C_{1}, C_{2}, C_{3}$ is not demonstrated in this paper. Such a choice can be made only if the functions $F$ and $G$ satisfy suitable restrictions which do not seem to be easy to characterize explicitly. In the following sections it is shown that for special examples the method can be carried through and the most general integral with a given family of extremals determined.

\section{INTEGRALS WHOSE EXTREMALS ARE STRAIGHT LINES}

The example of this section is inserted chiefly to show the application of the above theory to the problem which has already been treated by Hàmel.*

Consider the equations

$$
y^{\prime \prime}=0, \quad z^{\prime \prime}=0 .
$$

If these are the solutions for $y^{\prime \prime}$ and $z^{\prime \prime}$ of two equations

$$
H\left(x, y, z, y^{\prime}, z^{\prime}, y^{\prime \prime}, z^{\prime \prime}\right)=0, \quad K\left(x, y, z, y^{\prime}, z^{\prime}, y^{\prime \prime}, z^{\prime \prime}\right)=0
$$

whose equations of variation are a self-adjoint system, then there exists a set of multipliers $P, Q, R$ such that the functions $H$ and $K$ take the form

$$
H=P y^{\prime \prime}+Q z^{\prime \prime}=0, \quad K=Q y^{\prime \prime}+R z^{\prime \prime}=0 .
$$

The system (32) of Theorem $\mathrm{V}$ for this problem becomes the following:

$$
\begin{aligned}
P_{z^{\prime}}=Q_{y^{\prime}}, \quad Q_{z^{\prime}}=R_{y^{\prime}}, \\
P_{x}+P_{y} y^{\prime}+P_{z} z^{\prime}=0, \\
Q_{x}+Q_{y} y^{\prime}+Q_{z} z^{\prime}=0, \\
R_{x}+R_{y} y^{\prime}+R_{z} z^{\prime}=0,
\end{aligned}
$$

which must be identities in $x, y, z, y^{\prime}, z^{\prime}$. The last equation of (32) is identically zero since $F \equiv G \equiv 0$. Differentiate the third of equations (39) with respect to $z^{\prime}$ and the fourth with respect to $y^{\prime}$ and subtract. Proceed similarly with the fourth and fifth equations. Then, with the aid of the first two relations of (39), we have

\footnotetext{
* G. Hamel, Die Geometrieen im Raume, Mathematische Annalen, vol. 57, p. 255 ff.
} 


$$
P_{z}=Q_{y}, \quad Q_{z}=R_{y} .
$$

It is evident from the preceding section that, as a preliminary to the solving of the last three equations of the system (39), we must solve the equations

$$
y^{\prime \prime}=0, \quad z^{\prime \prime}=0 .
$$

By integration we find the equations

$$
\begin{aligned}
y^{\prime} & =a_{1}, & z^{\prime} & =a_{2}, \\
y & =a_{1} x+a_{3}, & z & =a_{2} x+a_{4},
\end{aligned}
$$

analogous to the equations (33); where $a_{1}, a_{2}, a_{3}, a_{4}$ are constants of integration. The values of $a_{1}, a_{2}, a_{3}, a_{4}$ in terms of $x, y, z, y^{\prime}, z^{\prime}$ found by solving the above equations are

$$
\begin{array}{ll}
a_{1}=y^{\prime}, & a_{2}=z^{\prime}, \\
a_{3}=y-y^{\prime} x, & a_{4}=z-z^{\prime} x,
\end{array}
$$

which correspond to the values (34) in the general case.

The equations corresponding to (35) are the following:

$$
\frac{d P}{d x}=0, \frac{d Q}{d x}=0, \frac{d R}{d x}=0 .
$$

Hence, the solutions of the last three equations of (39) are arbitrary functions

$$
P\left(a_{1}, a_{2}, a_{3}, a_{4}\right), \quad Q\left(a_{1}, a_{2}, a_{3}, a_{4}\right), \quad R\left(a_{1}, a_{2}, a_{3}, a_{4}\right)
$$

of the functions $a_{1}, a_{2}, a_{3}, a_{4}$.

Thus necessary and sufficient conditions for the system (38) to be selfadjoint are

$$
\begin{gathered}
P_{z^{\prime}}=Q_{y^{\prime}}, \quad Q_{z^{\prime}}=R_{y^{\prime}}, \\
P=P\left(a_{1}, a_{2}, a_{3}, a_{4}\right), \quad Q=Q\left(a_{1}, a_{2}, a_{3}, a_{4}\right), \\
R=R\left(a_{1}, a_{2}, a_{3}, a_{4}\right),
\end{gathered}
$$

in which $a_{1}, a_{2}, a_{3}, a_{4}$ have the values (41). One can readily show that the functions $P, Q, R$ can be determined so that the first two of relations (42) are satisfied.

To do this let $P, Q, R$ be differentiated with respect to $a_{1}, \cdots, a_{4}$, and these in turn differentiated with respect to $y^{\prime}$ and $z^{\prime}$. We have for the first and second of (42) respectively

$$
\begin{aligned}
& P_{2}-P_{4} x \equiv Q_{1}-Q_{3} x, \\
& Q_{2}-Q_{4} x \equiv R_{1}-R_{3} x .
\end{aligned}
$$


Regarding these equations as identities in $x$ we must have

$$
\begin{array}{ll}
P_{2}=Q_{1}, & P_{4}=Q_{3}, \\
Q_{2}=R_{1}, & Q_{4}=R_{3} .
\end{array}
$$

If the first equation is differentiated with respect to $a_{4}$ and the second with respect to $a_{2}$, we see that the cwo equations are compatible if

$$
Q_{14}=Q_{32} \text {. }
$$

Similarly, differentiating the last two equations with respect to $a_{3}, a_{1}$, respectively, we arrive at the same condition. Hence, there exist values of $P$, $Q, R$ which satisfy this system. It follows that the necessary and sufficient conditions (42) for self-adjointness of equations (38) can be satisfied.

If a value of $Q$ is selected satisfying the last equation, then by (43) $P$ is determined to an additive function of $a_{1}, a_{3}$, and $R$ is determined to an additive function of $a_{2}, a_{4}$. In the functions thus determined for $P, Q, R$ let $a_{1}, \cdots, a_{4}$ be replaced by their respective values in (41); the resulting functions satisfy the self-adjoint relations (39). Using these values we find a particular solution of the system

$$
\begin{aligned}
& g_{y^{\prime} y^{\prime}}=P\left(y^{\prime}, z^{\prime}, y-y^{\prime} x, z-z^{\prime} x\right), \\
& g_{y^{\prime} z^{\prime}}=Q\left(y^{\prime}, z^{\prime}, y-y^{\prime} x, z-z^{\prime} x\right), \\
& g_{z^{\prime} z^{\prime}}=R\left(y^{\prime}, z^{\prime}, y-y^{\prime} x, z-z^{\prime} x\right) .
\end{aligned}
$$

Since the conditions for integrability of this system are the first two of relations (39), which also insure that the following integrals are independent of the path of integration, the value of $g$ is given by the double integral

$$
g=\int_{y 0^{\prime} z_{0^{\prime}}}^{y^{\prime} z^{\prime}} \int_{y_{0^{\prime}} z_{0^{\prime}}}^{y^{\prime} z^{\prime}} P d y^{\prime} d y^{\prime}+2 Q d y^{\prime} d z^{\prime}+R d z^{\prime} d z^{\prime} .
$$

Corresponding to the line integrals $M$ and $L$ of $\S I I I$, we have

$$
g_{y^{\prime}}=\int_{y_{0^{\prime} z_{0}^{\prime}}^{y^{\prime}}}^{y^{\prime} z^{\prime}} P d y^{\prime}+Q d z^{\prime}, \quad g_{z^{\prime}}=\int_{y_{0^{\prime} z z^{\prime}}^{y^{\prime} z^{\prime}}}^{y^{\prime}} Q d y^{\prime}+R d z^{\prime} .
$$

Applying relations (40), which were shown to be consequences of (39), to the last two integrals we get

$$
g_{y^{\prime} z}=g_{z^{\prime} y} .
$$

By means of this relation and the fact that the functions $M$ and $N$ of (26) are each identically zero, equations (21) of \$III become

$$
\begin{aligned}
& c_{y}-b_{z}=0, \\
& a_{z}-c_{x}=-g_{z}+g_{z^{\prime} x}+g_{y^{\prime} z} y^{\prime}+g_{z^{\prime} z} z^{\prime}, \\
& b_{x}-a_{y}=g_{y}-g_{y^{\prime} x}-g_{y^{\prime} y} y^{\prime}-g_{z^{\prime} y} z^{\prime} .
\end{aligned}
$$


It is readily verified that this system satisfies the required conditions (22) which insure the existence of solutions for $a, b, c$.

In this particular case it is easy to show that, on account of relations (40), the second members of the last two equations vanish identically. By means of the integrals given above for $g$ and $g_{v^{\prime}}$ we have

$$
\begin{aligned}
& g_{y}-g_{y^{\prime} x}-g_{y^{\prime} y} y^{\prime}-g_{y^{\prime} z} z^{\prime}=\int_{y_{0}^{\prime} z_{0^{\prime}}^{\prime}}^{y^{\prime} z^{\prime}} \int_{y_{0^{\prime}} z_{0^{\prime}}}^{y^{\prime} z^{\prime}} P_{y} d y^{\prime} d y^{\prime}+2 Q_{y} d y^{\prime} d z^{\prime}+R_{y} d z^{\prime} d z^{\prime} \\
& -\int_{y_{0^{\prime}} z_{0^{\prime}}}^{y^{\prime} z^{\prime}} P_{x} d y^{\prime}+Q_{x} d z^{\prime}-y^{\prime} \int_{y_{0^{\prime}} z_{0^{\prime}}}^{y^{\prime} z^{\prime}} P_{y} d y^{\prime}+Q_{y} d z^{\prime}-z^{\prime} \int_{y_{0^{\prime}} z_{0^{\prime}}}^{y^{\prime} z^{\prime}} P_{s} d y^{\prime}+Q_{x} d z^{\prime} .
\end{aligned}
$$

If in the second integral the values of $P_{x}$ and $Q_{x}$ obtained from (39) are inserted, we have

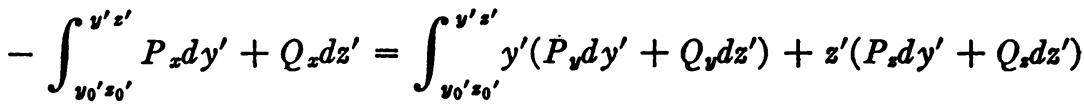

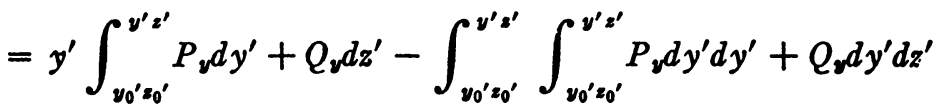

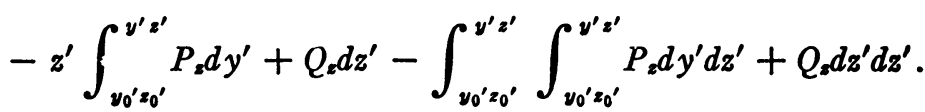

When this value of the integral on the left is substituted in the previous equation the result is identically zero in view of the relations (40). The second member of $\left(46_{2}\right)$ may be treated similarly. Hence, we have the conditions

$$
c_{y}-b_{z}=0, \quad a_{z}-c_{x}=0, \quad b_{x}-a_{y}=0 .
$$

Therefore, the integrand $f$ of the integral whose extremals are straight lines is of the form

$$
f=g\left(x, y, z, y^{\prime}, z^{\prime}\right)+\frac{d}{d x} t(x, y, z),
$$

where $g$ is a particular solution of the system (44) and $t$ is an arbitrary function of $x, y, z$.

VI. INTEgRALS WHOSE EXTREMALS ARE SEMT-CIRCLES ORTHOGONAL TO THE $x y$-PLANE

Consider the four-parameter family of circles

$$
\begin{aligned}
& x=a+b \cos d \cos u, \\
& y=c-b \sin d \cos u, \\
& z=b \sin u,
\end{aligned}
$$


where $u$ is the angle which the radius vector makes with the line of intersection of the plane of the circle and the $x y$-plane, and $d$ is the angle which this line of intersection makes with the positive $x$-axis.

The differential equations of these curves found by eliminating the parameter $u$ and the four constants $a, b, c, d$ are

$$
y^{\prime \prime}=0, \quad z^{\prime \prime}=-\frac{1+y^{\prime 2}+z^{\prime 2}}{z} .
$$

When the second members of these equations are substituted for $F$ and $G$ in the system (32) and the independent variables $x, y, z, y^{\prime}, z^{\prime}$ replaced by $x, a, b, c, d$ this system takes the form

$$
\begin{aligned}
& P_{z^{\prime}}=Q_{y^{\prime}}, \quad Q_{z^{\prime}}=R_{y^{\prime}}, \\
& \frac{d P}{d x}=\frac{2 Q y^{\prime}}{z},
\end{aligned}
$$

The coefficients in the last equation of (32) for this problem all vanish identically on account of the form of $F$ and $G$.

The integrals of equations (48) are the curves (47). When the variable $u$ is eliminated from the equations (47) and the derivatives $y^{\prime}, z^{\prime}$ with respect to $x$ are calculated we find

$$
\begin{aligned}
y=c+(a-x) \tan d, & z=\sec d\left(b^{2} \cos ^{2} d-(x-a)^{2}\right)^{1 / 2}, \\
y^{\prime}=-\tan d, & z^{\prime}=\frac{\sec d(a-x)}{\left(b^{2} \cos ^{2} d-(x-a)^{2}\right)^{1 / 2}} .
\end{aligned}
$$

When these are solved for $a, b, c, d$ the resulting functions

$$
\begin{aligned}
& a=x+\frac{z z^{\prime}}{1+y^{\prime 2}}, \quad b^{2}=z^{2}+\frac{z^{2} z^{\prime 2}}{1+y^{\prime 2}}, \\
& c=y+\frac{z y^{\prime} z^{\prime}}{1+y^{\prime 2}}, \quad \tan d=-y^{\prime}
\end{aligned}
$$

are four independent solutions of the homogeneous equation

$$
A_{x}+A_{y} y^{\prime}+A_{z} z^{\prime}+A_{y^{\prime}} 0-A_{z^{\prime}} \frac{1+y^{\prime 2}+z^{\prime 2}}{z} \equiv 0
$$

which characterizes the first integral of equations (48). But if (50) are solutions of this equation then also is any function of them a solution and in particular the following:

$$
\begin{array}{ll}
a_{1}=c+a \tan d, & a_{2}=a-c \tan d, \\
a_{3}=b^{2} \sec ^{2} d, & a_{4}=-\tan d
\end{array}
$$


which are selected because of their simplicity in terms of the variables $x$, $y, z, y^{\prime}, z^{\prime}$. In terms of these variables they have the values

$$
\begin{array}{ll}
a_{1}=y-y^{\prime} x, & a_{2}=x+y y^{\prime}+z z^{\prime}, \\
a_{3}=z\left(1+y^{\prime 2}+z^{\prime 2}\right), . & a_{4}=y^{\prime} .
\end{array}
$$

It is evident from the form of equations (49) that the last one can be integrated directly and, hence, also the second and the first. The general solution of the system is

$$
\begin{array}{lr}
P=\alpha+2 \beta y+\gamma y^{2}, \\
Q= & \beta z+\gamma y^{2}, \\
R= & \gamma z^{2},
\end{array}
$$

where $\alpha, \beta, \gamma$ are constants of integration and arbitrary functions of $a_{1}$, $a_{2}, a_{3}, a_{4}$.

With the values of $F$ and $G$ given in (48) it is readily verified, as has been mentioned above, that the coefficients of $P, Q, R$ in the last equation of (32) are each identically zero. This is not a characteristic property of the functions $F$ and $G$ for every such problem of the calculus of variations, though it holds for this particular case.

It remains now to show that there exist values of $\alpha, \beta, \gamma$ such that the remaining conditions for self-adjointness, namely

$$
P_{z^{\prime}}=Q_{y^{\prime}}, \quad Q_{z^{\prime}}=R_{y^{\prime}},
$$

are satisfied. Imposing these conditions on the second members in (52), we have

$$
\begin{aligned}
& y^{2} \gamma_{z^{\prime}}+2 y \beta_{z^{\prime}}+\alpha_{z^{\prime}} \equiv y z \gamma_{y^{\prime}}+z \beta_{y^{\prime}} \text {, } \\
& y \gamma_{z^{\prime}}+\beta_{z^{\prime}} \equiv z \gamma_{y^{\prime}} \text {, }
\end{aligned}
$$

which after a simple recombination are equivalent to the following:

$$
\begin{aligned}
& z \gamma_{y^{\prime}}-y \gamma_{z^{\prime}}=\beta_{z^{\prime}}, \\
& z \beta_{y^{\prime}}-y \beta_{z^{\prime}}=\alpha_{z^{\prime}} .
\end{aligned}
$$

After differentiating $\alpha, \beta, \gamma$ with respect to $a_{1}, a_{2}, a_{3}, a_{4}$ and these in turn, as given in (51), with respect to $y^{\prime}$ and $z^{\prime}$, equations (53) become

$$
\begin{aligned}
& -x \gamma_{1}+2 z\left(y^{\prime} z-y z^{\prime}\right) \gamma_{3}+\gamma_{4}-\beta_{2}-2 z z^{\prime} \beta_{3}=0, \\
& -x \beta_{1}+2 z\left(y^{\prime} z-y z^{\prime}\right) \beta_{3}+\beta_{4}-\alpha_{2}-2 z z^{\prime} \alpha_{3}=0,
\end{aligned}
$$

in which the subscripts $1,2,3,4$ denote differentiation with respect to $a_{1}, a_{2}$, $a_{3}, a_{4}$ respectively. If equations (51) are solved for $y, z, y^{\prime}, z^{\prime}$ and their values, namely 


$$
\begin{aligned}
y & =a_{1}+a_{4} x, & z & =\left(\frac{a_{3}-\left[\left(1+a_{4}^{2}\right) x+\left(a_{1} a_{4}-a_{2}\right)\right]^{2}}{1+a_{4}^{2}}\right)^{1 / 2}, \\
y^{\prime} & =a_{4}, & z^{\prime} & =\left(\frac{\left(1+a_{4}^{2}\right)\left[a_{2}-a_{1} a_{4}-\left(1+a_{4}^{2}\right) x\right]^{2}}{a_{3}-\left[\left(1+a_{4}^{2}\right)+\left(a_{1} a_{4}-a_{2}\right)\right]^{2}}\right)^{1 / 2},
\end{aligned}
$$

are substituted in equations (54), the resulting equations must be identities in $x, a_{1}, \cdots, a_{4}$. This substitution is performed in the first equation of (54) and the coefficients collected according to the powers of $x$. The coefficient of $x^{2}$ vanishes; that of $x$ and the constant term give the first two of the following conditions. Similar results from the second equation of (54) follow by symmetry and give the last two conditions:

$$
\begin{gathered}
2\left(a_{1}+a_{2} a_{4}\right) \gamma_{3}+2\left(1+a_{4}^{2}\right) \beta_{3}-\gamma_{1}=0, \\
2\left[a_{4} a_{3}-\left(a_{1}+a_{2} a_{4}\right)\left(a_{2}-a_{1} a_{4}\right)\right] \gamma_{3}-2\left(a_{2}-a_{1} a_{4}\right)\left(1+a_{4}^{2}\right) \beta_{3} \\
+\left(1+a_{4}^{2}\right) \gamma_{4}-\left(1+a_{4}^{2}\right) \beta_{2}=0, \\
2\left(a_{1}+a_{2} a_{4}\right) \beta_{3}+2\left(1+a_{4}^{2}\right) \alpha_{3}-\beta_{1}=0, \\
2\left[a_{4} a_{3}-\left(a_{1}+a_{2} a_{4}\right)\left(a_{2}-a_{1} a_{4}\right)\right] \beta_{3}-2\left(a_{2}-a_{1} a_{4}\right)\left(1+a_{4}^{2}\right) \alpha_{3} \\
+\left(1+a_{4}^{2}\right) \beta_{4}-\left(1+a_{4}^{2}\right) \alpha_{2}=0 .
\end{gathered}
$$

The first two of equations (55) can be solved for $\beta_{2}$ and $\beta_{3}$. One obtains

$$
\begin{aligned}
& \left(1+a_{4}^{2}\right) \beta_{2}=-\left(a_{9}-a_{1} a_{4}\right) \gamma_{1}+2 a_{3} a_{4} \gamma_{3}+\left(1+a_{4}^{2}\right) \gamma_{4}, \\
& \left(1+a_{4}^{2}\right) \beta_{3}=\frac{1}{2} \gamma_{1}-\left(a_{1}+a_{2} a_{4}\right) \gamma_{3} .
\end{aligned}
$$

If these equations are differentiated with respect to $a_{3}$ and $a_{4}$ respectively, then

$$
\begin{aligned}
& \left(1+a_{4}^{2}\right) \beta_{23}=-\left(a_{2}-a_{1} a_{4}\right) \gamma_{13}+2 a_{3} a_{4} \gamma_{33}+2 a_{4} \gamma_{3}+\left(1+a_{4}^{2}\right) \gamma_{43} . \\
& \left(1+a_{4}^{2}\right) \beta_{32}=\frac{1}{2} \gamma_{12}-\left(a_{1}+a_{2} a_{4}\right) \gamma_{32}-a_{4} \gamma_{3} .
\end{aligned}
$$

If the equations (56) are consistent the second members of (57) must be identical. Hence, we have the condition

$$
\begin{aligned}
3 a_{4} \gamma_{3}=\left(a_{2}-a_{1} a_{4}\right) \gamma_{13} & -\left(a_{1}+a_{2} a_{4}\right) \gamma_{32}-2 a_{3} a_{4} \gamma_{33} \\
- & \left(1+a_{4}^{2}\right) \gamma_{43}+\frac{1}{2} \gamma_{12}
\end{aligned}
$$

that must be satisfied by the function $\gamma$. A pair of equations similar to (56) for $\alpha$ and $\beta$ is obtained from the last two equations of (55). They are, in fact, the system (56) with $\beta, \gamma$ replaced by $\alpha, \beta$ respectively. These new equations are compatible only if $\beta$ satisfies the equation obtained from (58) by replacing $\gamma$ by $\beta$. If in this equation for $\beta$ its derivatives with respect 
to $a_{1}, a_{2}, a_{3}, a_{4}$ are replaced by their respective values obtained from (56) and (57) the result again reduces to the condition (58).

Therefore, equations (53) are consistent and there exist values of the functions $\alpha, \beta, \gamma$ such that the conditions

$$
P_{z^{\prime}}=Q_{y^{\prime}}, \quad Q_{z^{\prime}}=R_{y^{\prime}}
$$

are satisfied provided that $\gamma$ satisfies equation (58).

ThEorem VI. The most general integral

$$
I=\int_{x_{1}}^{x_{2}} f\left(x, y, z, y^{\prime}, z^{\prime}\right) d x
$$

whose extremals are the family of circles (47) satisfying the equation (48) is determined as follows:

For an arbitrary solution $\gamma\left(a_{1}, a_{2}, a_{3}, a_{4}\right)$ of the equation (58) the equations (56) are compatible and determine $\beta\left(a_{1}, a_{2}, a_{3}, a_{4}\right)$ to an arbitrary additive function of $a_{1}, a_{4}$. Equations (56) with $\gamma$ replaced by this function $\beta$ and $\beta$ replaced by $\alpha$, are again compatible and define $\alpha\left(a_{1}, a_{2}, a_{3}, a_{4}\right)$ to an arbitrary additive function of $a_{1}, a_{4}$. The functions

$$
\begin{array}{lr}
P\left(x, y, z, y^{\prime}, z^{\prime}\right)=\alpha+2 \beta y+\gamma y^{2}, \\
Q\left(x, y, z, y^{\prime}, z^{\prime}\right)= & \beta z+\gamma y^{2}, \\
R\left(x, y, z, y^{\prime}, z^{\prime}\right)= & \gamma z^{2}
\end{array}
$$

so determined satisfy the conditions (49) which are necessary and sufficient for the equations

$$
\begin{aligned}
& H=P y^{\prime \prime}+Q\left(z^{\prime \prime}+\frac{1+y^{\prime 2}+z^{\prime}}{z}\right)=0 \\
& K=Q y^{\prime \prime}+R\left(z^{\prime \prime}+\frac{1+y^{\prime 2}+z^{\prime 2}}{z}\right)=0
\end{aligned}
$$

to have equations of variation which are self-adjoint. By the process of \$III the integrand function $f\left(x, y, z, y^{\prime}, z^{\prime}\right)$ is then determined to an additive derivative of an arbitrary function of $x, y, z$. The arbitrary elements are, therefore, the choice of an integral of the partial differential equation (58) of the second order, the choice of two arbitrary additive functions of $a_{1}$ and $a_{4}$, and the choice of the derivative of an arbitrary additive function of $(x, y, z)$. 
VII. INTEGRALS WHOSE EXTREMALS ARE CATENARIES ORTHOGONAL TO THE $x y$-PLANE

Consider the four-parameter family of catenaries

$$
\begin{aligned}
& x=a+c \cos \theta \cosh ^{-1}\left(\frac{s^{2}+c^{2}}{c}\right)^{1 / 2}, \\
& y=b-c \sin \theta \cosh ^{-1}\left(\frac{s^{2}+c^{2}}{c}\right)^{1 / 2}, \\
& z=\left(s^{2}+c^{2}\right)^{1 / 2},
\end{aligned}
$$

where $s$ is the arc length of the catenary measured from the lowest point and $\theta$ is the angle which the line of intersection of the plane of the catenary and of the $x y$-plane makes with the positive $x$-axis.

The differential equations of the curves (1) found by eliminating the parameter $s$ and the four constants $a, b, c, \theta$ are

$$
y^{\prime \prime}=0, \quad z^{\prime \prime}=\frac{1+y^{\prime 2}+z^{\prime 2}}{z} .
$$

If these values of $y^{\prime \prime}$ and $z^{\prime \prime}$ are substituted for $F$ and $G$ respectively in the system (32), §IV, the latter take the following form:

$$
\begin{aligned}
P_{z^{\prime}} & =Q_{y^{\prime}}, \quad Q_{z^{\prime}}=R_{y^{\prime}}, \\
\frac{d P}{d x}=-Q \frac{2 y^{\prime}}{z}, & \frac{d Q}{d x}=-\left(Q \frac{z^{\prime}}{z}+R \frac{y^{\prime}}{z}\right), \frac{d R}{d x}=-R \frac{2 z^{\prime}}{z}, \\
Q\left(1+y^{\prime 2}\right)+R y^{\prime} z^{\prime} & =0 .
\end{aligned}
$$

The integrals of (60) are the curves (59). When the parameter $s$ is eliminated from the equations (59) and the derivatives $y^{\prime}$ and $z^{\prime}$ are found, one obtains

$$
\begin{array}{rlrl}
y & =b-(x-a) \tan \theta, & z & =c \cosh \left(\frac{x-a}{c \cos \theta}\right) \\
y^{\prime} & =-\tan \theta, & z^{\prime}=\sec \theta \sinh \left(\frac{x-a}{c \cos \theta}\right) .
\end{array}
$$

If the substitutions

$$
a_{1}=-\tan \theta, \quad a_{2}=b, \quad a_{3}=c, \quad a_{4}=a
$$

are made equations (62) become 


$$
\begin{array}{ll}
y=a_{2}-a_{1} a_{4}+a_{1} x, \quad z=a_{3} \cosh \left(\frac{\left(x-a_{4}\right)\left(1+a_{1}^{2}\right)^{1 / 2}}{a_{3}}\right), \\
y^{\prime}=a_{1}, & \quad \cdot=\left(1+a_{1}^{2}\right)^{1 / 2} \sinh \frac{\left(x-a_{4}\right)\left(1+a_{1}^{2}\right)^{1 / 2}}{a_{3}} .
\end{array}
$$

When these equations are solved for $a_{1}, a_{2}, a_{3}, a_{4}$ the resulting functions

$$
\begin{aligned}
& a_{1}=y^{\prime}, \quad a_{2}=y-\frac{y_{z}^{\prime}}{\left(1+y^{\prime 2}+z^{\prime 2}\right)^{1 / 2}} \cosh ^{-1}\left(\frac{1+y^{\prime 2}+z^{\prime 2}}{1+y^{\prime 2}}\right)^{1 / 2}, \\
& a_{8}=\frac{z\left(1+y^{\prime 2}\right)^{1 / 2}}{\left(1+y^{\prime 2}+z^{\prime 2}\right)^{1 / 2}}, \\
& a_{4}=x-\frac{z}{\left(1+y^{\prime 2}+z^{\prime 2}\right)^{1 / 2}} \cosh ^{-1}\left(\frac{1+y^{\prime 2}+z^{\prime 2}}{1+y^{\prime 2}}\right)^{1 / 2}
\end{aligned}
$$

are four independent solutions of the homogeneous equation

$$
A_{x}+A_{y} y^{\prime}+A_{z} z^{\prime}+A_{y^{\prime}} 0+A_{z^{\prime}} \frac{1+y^{\prime 2}+z^{\prime 2}}{z}=0,
$$

which characterizes the first integral of equations (60).

The fifth equation of the system (61) can be integrated directly and then also the fourth and the third. They yield the following system:

$$
\begin{aligned}
& P=\alpha-\frac{2 \beta y^{\prime} z^{\prime}}{z\left(1+y^{\prime 2}\right)}-\frac{\gamma y^{\prime 2}}{z^{2}\left(1+y^{\prime 2}\right)}, \\
& Q=\frac{\beta}{z}-\frac{\gamma y^{\prime} z^{\prime}}{z^{2}\left(1+y^{\prime 2}\right)}, \\
& R=\frac{\gamma}{z^{2}}
\end{aligned}
$$

where $\alpha, \beta, \gamma$ are arbitrary functions of $a_{1}, \cdots, a_{4}$. The above values of $P, Q, R$ must satisfy the last of equations (61). We thus find that $\beta$ must be identically zero. The system (65) now becomes

$$
\begin{aligned}
& P=\alpha-\frac{\gamma y^{\prime 2}}{z^{2}\left(1+y^{\prime 2}\right)}, \\
& Q=-\frac{\gamma y^{\prime} z^{\prime}}{z^{2}\left(1+y^{\prime 2}\right)}, \\
& R=\frac{\gamma}{z^{2}} .
\end{aligned}
$$


If these functions are to satisfy the first two relations of (61) we must have the following conditions satisfied by $\alpha$ and $\gamma$ :

$$
\begin{aligned}
\frac{z^{\prime}\left(y^{\prime 2}-1\right) \gamma}{1+y^{\prime 2}}-y^{\prime} z^{\prime} \gamma_{y^{\prime}}+y^{\prime 2} \gamma_{z^{\prime}}=z^{2}\left(1+y^{\prime 2}\right) \alpha_{z^{\prime}}, \\
y^{\prime} \gamma+\left(1+y^{\prime 2}\right) \gamma_{y^{\prime}}+y^{\prime} z^{\prime} \gamma_{z^{\prime}}=0,
\end{aligned}
$$

which shall be identities in $x, a_{1}, \cdots, a_{4}$. These equations are equivalent to the system

$$
\begin{aligned}
z^{\prime}\left(2 y^{\prime 2}-1\right) \gamma+y^{\prime 2}\left(1+y^{\prime 2}+z^{\prime 2}\right) \gamma_{z^{\prime}} & =z^{2}\left(1+y^{\prime 2}\right)^{2} \alpha_{z^{\prime}} \\
y^{\prime} \gamma+\left(1+y^{\prime 2}\right) \gamma_{z^{\prime}}+y^{\prime} z^{\prime} \gamma_{z^{\prime}} & =0 .
\end{aligned}
$$

Since $\alpha, \gamma$ are functions of $a_{1}, \cdots, a_{4}$ they may be differentiated with respect to $a_{1}, \cdots, a_{4}$ and these functions in turn differentiated with respect to $y^{\prime}$ and $z^{\prime}$. Thus equations (67) give the following relations:

$$
\begin{gathered}
\gamma y^{\prime}+\gamma_{1}\left(1+y^{\prime 2}\right)-\gamma_{2} \frac{z \cosh ^{-1} u}{\left(1+y^{\prime 2}+z^{\prime 2}\right)^{1 / 2}}+\gamma_{3} \cdot 0+\gamma_{4} \frac{z y^{\prime} \cosh ^{-1} u}{\left(1+y^{\prime 2}+z^{\prime 2}\right)^{1 / 2}}=0 \\
\gamma z^{\prime}\left(2 y^{\prime 2}-1\right)+\gamma_{2}\left(\frac{z y^{\prime 3} z^{\prime} \cosh ^{-1} u}{\left(1+y^{\prime 2}+z^{\prime 2}\right)^{1 / 2}}-z y^{\prime 3}\right)-\gamma_{3} \frac{z y^{\prime 2} z^{\prime}\left(1+y^{\prime 2}\right)^{1 / 2}}{\left(1+y^{\prime 2}+z^{\prime 2}\right)^{1 / 2}} \\
+\gamma_{4} \frac{z y^{\prime 2} z^{\prime} \cosh ^{-1} u-z y^{\prime 2}}{\left(1+y^{\prime 2}+z^{\prime 2}\right)^{1 / 2}} \\
=\alpha_{2}\left[\frac{z^{3} y^{\prime} z^{\prime}\left(1+y^{\prime 2}\right)^{2} \cosh ^{-1} u}{\left(1+y^{\prime 2}+z^{\prime 2}\right)^{3 / 2}}-\frac{y^{\prime} z^{3}\left(1+y^{\prime 2}\right)^{2}}{\left(1+y^{\prime 2}+z^{\prime 2}\right)}\right] \\
-\alpha_{3} \frac{z^{3} z^{\prime}\left(1+y^{\prime 2}\right)^{5 / 2}}{\left(1+y^{\prime 2}+z^{\prime 2}\right)^{3 / 2}}+\alpha_{4}\left[\frac{z^{3} z^{\prime}\left(1+y^{\prime 2}\right)^{2} \cosh ^{-1} u}{\left(1+y^{\prime 2}+z^{\prime 2}\right)^{3 / 2}}-\frac{z^{3}\left(1+y^{\prime 2}\right)^{2}}{\left(1+y^{\prime 2}+z^{\prime 2}\right)}\right]
\end{gathered}
$$

where the subscripts $1,2,3,4$ indicate differentiation with respect to $a_{1}$, $a_{2}, a_{3}, a_{4}$ respectively and

$$
u=\left(\frac{1+y^{\prime 2}+z^{\prime 2}}{1+y^{\prime 2}}\right)^{1 / 2} .
$$

If the values given in (63) for $y, z, y^{\prime}, z^{\prime}$ respectively are substituted in (68), one obtains

$$
a_{1} \gamma+\left(1+a_{1}^{2}\right) \gamma_{1}-\left(x-a_{4}\right) \gamma_{2}+a_{1}\left(x-a_{4}\right) \gamma_{1}=0 \text {, }
$$

(69) $\gamma\left(1+a_{1}^{2}\right)^{1 / 2}\left(2 a_{1}^{2}-1\right) \sinh \omega+\gamma_{2} a_{1}^{3}\left[\left(x-a_{4}\right)\left(1+a_{1}^{2}\right)^{1 / 2} \sinh \omega-a_{3} \cosh \omega\right]$

$-\gamma_{3} a_{1}^{2} a_{3}\left(1+a_{1}^{2}\right)^{1 / 2} \sinh \omega+\gamma_{4} a_{1}^{3}\left[\left(x-a_{4}\right)\left(1+a_{1}^{2}\right)^{1 / 2} \sinh \omega-a_{3} \cosh \omega\right]$ $=\alpha_{2} a_{1} a_{3}^{2}\left(1+a_{1}^{2}\right)\left[\left(x-a_{4}\right)\left(1+a_{1}^{2}\right)^{1 / 2} \sinh \omega-a_{3} \cosh \omega\right]$

$-\alpha_{3} a_{3}^{3}\left(1+a_{1}^{2}\right)^{3 / 2} \sinh \omega+\alpha_{4} a_{3}^{2}\left(1+a_{1}^{2}\right)\left[\left(x-a_{4}\right)\left(1+a_{1}^{2}\right)^{1 / 2} \sinh \omega\right.$ $\left.-a_{3} \cosh \omega\right]$ 
in which

$$
\omega=\frac{\left(x-a_{4}\right)\left(1+a_{1}^{2}\right)^{1 / 2}}{a_{3}} .
$$

The last equation may be written in the form

$$
\begin{aligned}
& {\left[\left(1+a_{1}^{2}\right)^{1 / 2}\left(2 a_{1}^{2}-1\right) \gamma-a_{1}^{3} a_{4}\left(1+a_{1}^{2}\right)^{1 / 2} \gamma_{2}-a_{1}^{2} a_{3}\left(1+a_{1}^{2}\right)^{1 / 2} \gamma_{3}\right.} \\
& -a_{1}^{2} a_{4}\left(1+a_{1}^{2}\right)^{1 / 2} \gamma_{4}+a_{1} a_{3}^{2} a_{4}\left(1+a_{1}^{2}\right)^{3 / 2} \alpha_{2}+a_{3}^{3}\left(1+a_{1}^{2}\right)^{3 / 2} \alpha_{3} \\
& \left.+a_{3}^{2} a_{4}\left(1+a_{1}^{2}\right)^{3 / 2} \alpha_{4}\right]+\left[a_{1}^{3}\left(1+a_{1}^{2}\right)^{1 / 2} \gamma_{2}+a_{1}^{2}\left(1+a_{1}^{2}\right)^{1 / 2} \gamma_{4}\right. \\
& \left.-a_{1} a_{3}^{2}\left(1+a_{1}^{2}\right)^{3 / 2} \alpha_{2}-a_{3}^{2}\left(1+a_{1}^{2}\right)^{3 / 2} \alpha_{4}\right] x-\left[a_{1}^{3} a_{3} \gamma_{2}+a_{1}^{2} a_{3} \gamma_{4}\right. \\
& \left.-a_{1} a_{3}^{8}\left(1+a_{1}^{2}\right) \alpha_{2}-a_{3}^{3}\left(1+a_{1}^{2}\right) \alpha_{4}\right] \operatorname{coth} \omega=0 .
\end{aligned}
$$

These equations must be identities in $x$. Hence, from the first of (69), we have

$$
a_{1} \gamma_{4}-\gamma_{2}=0, \quad a_{1} \gamma+\left(1+a_{1}^{2}\right) \gamma_{1}+a_{4} \gamma_{2}-a_{1} a_{4} \gamma_{4}=0,
$$

which are equivalent to the system

$$
a_{1} \gamma_{4}-\gamma_{2}=0, \quad a_{1} \gamma+\left(1+a_{1}^{2}\right) \gamma_{1}=0 .
$$

Equation $\left(69^{\prime}\right)$ upon equating the respective coefficients to zero gives

$$
\begin{aligned}
& a_{1}^{2}\left(a_{1} \gamma_{2}+\gamma_{4}\right)=a_{3}^{2}\left(1+a_{1}^{2}\right)\left(a_{1} \alpha_{2}+\alpha_{4}\right), \\
& \left(2 a_{1}^{2}-1\right) \gamma-a_{1}^{3} a_{4} \gamma_{2}-a_{1}^{2} a_{3} \gamma_{3}-a_{1}^{2} a_{4} \gamma_{4} \\
& =-a_{3}^{2}\left(1+a_{1}^{2}\right)\left(a_{1} a_{4} \alpha_{2}+a_{3} \alpha_{3}+a_{4} \alpha_{4}\right) .
\end{aligned}
$$

The latter are equivalent to the following relations:

$$
\begin{aligned}
a_{1}^{2}\left(a_{1} \gamma_{2}+\gamma_{4}\right) & =a_{3}^{2}\left(1+a_{1}^{2}\right)\left(a_{1} \alpha_{2}+\alpha_{4}\right), \\
\left(2 a_{1}^{2}-1\right) \gamma-a_{1}^{2} a_{3} \gamma_{3} & =-a_{3}^{3}\left(1+a_{1}^{2}\right) \alpha_{3} .
\end{aligned}
$$

When equations (71) are solved for $\alpha_{3}$ and $\alpha_{4}$ with the aid of the first of relations (70) one obtains

$$
\begin{aligned}
& \alpha_{4}=\frac{a_{1}^{2}}{a_{3}^{2}} \gamma_{4}-a_{1} \alpha_{2}, \\
& \alpha_{3}=\frac{-1}{a_{3}^{3}\left(1+a_{1}^{2}\right)}\left[\left(2 a_{1}^{2}-1\right) \gamma-a_{1}^{2} a_{3} \gamma_{3}\right] .
\end{aligned}
$$

If these equations are compatible the derivatives of the expressions on the right with respect to $a_{3}$ and $a_{4}$ respectively, after $\alpha_{23}$ in the first expression is replaced by its value obtained from the second, must be identically equal. With the aid of $\left(70_{1}\right)$ the result reduces to the condition

$$
\left(1+a_{1}^{2}\right) \gamma_{4}=0 \text {. }
$$

Hence, by the first equation of (70) $\gamma_{2}$ must also be identically. zero. Thus $\gamma$ 
must be a function of $a_{1}$ and $a_{3}$ alone such that it satisfies the second condition of (70). This will determine $\gamma$ to an additive function of $a_{3}$. From the above restriction on $\gamma$ and the first relation of (71) we have

$$
a_{1} \alpha_{2}+\alpha_{4}=0
$$

which together with the second equation of (71) will determine $\alpha$ to an additive function of $a_{1}$.

It is now evident that equations (67) are compatible and that there exist values of the functions $\gamma$ and $\alpha$ such that the relations

$$
P_{z^{\prime}}=Q_{y^{\prime}}, \quad Q_{z^{\prime}}=R_{y^{\prime}}
$$

are satisfied provided that $\gamma$ is a function of $a_{1}, a_{3}$ alone and satisfies the second equation of (70).

THEOREM VII. The most general integral

$$
I=\int_{x_{1}}^{x_{2}} f\left(x, y, z, y^{\prime}, z^{\prime}\right) d x
$$

whose extremals are the family of catenaries (59) satisfying equations (60) is determined as follows: For an arbitrary solution $\gamma\left(a_{1}, a_{3}\right)$ of the equation $\left(70_{1}\right)$ the equations (71) are compatible and determine $\alpha$ to an arbitrary additive function of $a_{1}$. The functions

$$
\begin{aligned}
& P=\alpha-\frac{\gamma y^{\prime 2}}{z^{2}\left(1+y^{\prime 2}\right)} \\
& Q=-\frac{\gamma y^{\prime} z^{\prime}}{z^{2}\left(1+y^{\prime 2}\right)} \\
& R=\frac{\gamma}{z^{2}}
\end{aligned}
$$

thus determined satisfy the conditions (61) which are necessary and sufficient for the equations

$$
\begin{aligned}
& H=P y^{\prime \prime}+Q\left(z^{\prime \prime}-\frac{1+y^{\prime 2}+z^{\prime 2}}{z}\right)=0 \\
& K=Q y^{\prime \prime}+R\left(z^{\prime \prime}-\frac{1+y^{\prime 2}+z^{\prime 2}}{z}\right)=0
\end{aligned}
$$

to have equations of variation which are self-adjoint. By the method of \$III the integrand function $f\left(x, y, z, y^{\prime}, z^{\prime}\right)$ is then determined to the derivative of an arbitrary function of $x, y, z$.

UNIVERSITY OF OREGON, EUGENE, Ore. 